\title{
Double Dissociation of the Effects of Medial and Orbital Prefrontal Cortical Lesions on Attentional and Affective Shifts in Mice
}

\author{
Gregory B. Bissonette, ${ }^{1,3 *}$ Gabriela J. Martins, ${ }^{1,3 *}$ Theresa M. Franz, ${ }^{1}$ Elizabeth S. Harper, ${ }^{1}$ Geoffrey Schoenbaum, ${ }^{1,2,3}$ \\ and Elizabeth M. Powell $1,2,3$ \\ Departments of ${ }^{1}$ Anatomy and Neurobiology and ${ }^{2}$ Psychiatry, and ${ }^{3}$ Program in Neuroscience, University of Maryland School of Medicine, Baltimore, \\ Maryland 21201
}

\begin{abstract}
Many neuropsychiatric diseases are associated with cognitive rigidity linked to prefrontal dysfunction. For example, schizophrenia and Parkinson's disease are associated with performance deficits on the Wisconsin Card Sorting Test, which evaluates attentional set shifting. Although the genetic underpinnings of these disorders can be reproduced in mice, there are few models for testing the functional consequences. Here, we demonstrate that an analog of the Wisconsin Card Sorting Test, developed in marmosets and recently adapted to rats, is a behavioral model of prefrontal function in mice. Systematic analysis demonstrated that formation of the attentional set in mice is dependent on the number of problem sets. We found that mice, like rats and primates, exhibit both affective and attentional sets, and these functions are disrupted by neurotoxic damage to orbitofrontal and medial prefrontal cortical areas, respectively. These data are identical to studies in rats and similar to the deficits reported after prefrontal damage in a comparable task in marmosets. These results provide a behavioral model to assess prefrontal function in mice.
\end{abstract}

Key words: executive function; cognitive flexibility; reversal learning; set shifting; Wisconsin Card Sorting Test; behavior

\section{Introduction}

Transgenic mouse models hold promise for elucidating the genetic basis of human neuropsychiatric disorders, including addiction, schizophrenia, autism, and degenerative disorders. These diseases often involve changes in cognitive flexibility that is dependent on prefrontal cortical areas (Shad et al., 2006; Verdejo-García et al., 2006; Clarke et al., 2007; Thoma et al., 2007). Currently, there are no good mouse models for testing prefrontal function. Here, we evaluated the rat reversal and setshifting task of Birrell and Brown (Birrell and Brown, 2000; Colacicco et al., 2002; McAlonan and Brown, 2003) for its suitability to test prefrontal cortical functioning in mice.

There is significant debate whether mice even exhibit key functions thought to be mediated by prefrontal areas in other species (Preuss, 1995; Uylings et al., 2003). For example, the ability to shift away from acquired attentional sets is dependent on

Received June 19, 2008; revised Sept. 8, 2008; accepted Sept. 12, 2008.

This work was supported by grants from University of Maryland Intramural Program (E.M.P.), a National Alliance for Research on Schizophrenia and Depression Young Investigator Award (E.M.P.), an Autism-Speaks Predoctoral Fellowship (G.J.M.), and National Institutes of Health Grants R01 AG027097 (G.S.), R01 DA018826, and R56 MH57683 (E.M.P.). We thank Mihyun Bae, David Jaffe, Tejas Suresh, Michelle Lande, and Michelle Harmon for participating in development of the behavioral task, and Drs. Thomas Stalnaker and Matthew Roesch for insightful discussions about our early results.

*G.B.B. and G.J.M. contributed equally to this work.

Correspondence should be addressed to Dr. Elizabeth M. Powell, Department of Anatomy and Neurobiology, University of Maryland School of Medicine, HSF II S251, 20 Penn Street, Baltimore, MD 21201. E-mail: epowe001@umaryland.edu or epowel@gmail.com.

DOI:10.1523/JNEUROSCI.2820-08.2008

Copyright $\odot 2008$ Society for Neuroscience $\quad 0270-6474 / 08 / 2811124-07 \$ 15.00 / 0$ prefrontal cortex (Dias et al., 1996a; Birrell and Brown, 2000; McAlonan and Brown, 2003; Clarke et al., 2004, 2005; Floresco et al., 2006). Although mice do seem to form affective sets, as evidenced by increased trials to acquire simple reversals (Colacicco et al., 2002; Lidow et al., 2003; Brigman et al., 2005; Glickstein et al., 2005; Izquierdo et al., 2006), initial attempts concluded that mice do not form attentional sets (Colacicco et al., 2002; Brigman et al., 2005). The addition of repetitive training (overtraining) (Garner et al., 2006) suggests that mice may be able to form attentional sets, although differently than rats and primates.

Frontal lobe lesions in the rat led to behavioral deficits comparable with those observed in primates (Kolb, 1984; Schoenbaum et al., 2003a; Uylings et al., 2003). Lesions to the medial prefrontal cortical (MFC) areas reduced formation of an attentional set, as measured by the Wisconsin Card Sorting Test in primates (Nelson, 1976; Dias et al., 1996a,b; Pantelis et al., 1999; Goldstein et al., 2004) or by the perceptual attentional setshifting task in rats (Birrell and Brown, 2000). Similar conclusions were drawn about orbital frontal cortical (OFC) regions (Schoenbaum et al., 2003a; Schoenbaum and Roesch, 2005). Lesions to the OFC region impaired goal-directed behaviors and reversal learning, whether the choices were presented as visual stimuli in the primate (O'Doherty et al., 2003; Remijnse et al., 2005) or rat (Chudasama and Robbins, 2003) or as odormediated rewards in the rat (McAlonan and Brown, 2003; Schoenbaum et al., 2003b). In summary, lesion studies have demonstrated correlations between structure and function of prefrontal cortical areas. 
Table 1. Order of discrimination tasks

\begin{tabular}{|c|c|c|c|c|}
\hline \multirow[b]{2}{*}{ Task } & \multicolumn{2}{|l|}{ Dimension } & \multicolumn{2}{|c|}{ Exemplar combinations } \\
\hline & Relevant $^{a}$ & Irrelevant & Correct & Incorrect \\
\hline SD & Odor & Medium & 01, M1 & $02, \mathrm{M} 1$ \\
\hline$C D$ & Odor & Medium & $01, M 1, M 2$ & $02, M 1, M 2$ \\
\hline IDS & Odor & Medium & $03, M 3, M 4$ & $04, M 3, M 4$ \\
\hline IDS || & Odor & Medium & 05, M5, M6 & 06, M5, M6 \\
\hline IDS III & Odor & Medium & 07, M7, M8 & 08, M7, M8 \\
\hline IDSIV & Odor & Medium & 09, M9, M10 & 010, M9, M10 \\
\hline IDS IVrev & Odor & Medium & 010, M9, M10 & 09, M9, M10 \\
\hline EDS & Medium & Odor & $M 11,011,012$ & $\mathrm{M} 12,011,012$ \\
\hline
\end{tabular}

M, Medium; 0, odor.

${ }^{a}$ The order of discriminations was the same for mice, but the relevant dimension, and thus the direction of the EDS (odor to medium or medium to odor) was counterbalanced within each experimental group. The number of trials required to reach criterion (eight correct consecutive trials) was independent of choice of relevant dimension.

The mouse literature lacks studies to show specific correlations between prefrontal regions and cognitive function. Using a modified reversal learning and set-shift task that was developed for the rat (Birrell and Brown, 2000; McAlonan and Brown, 2003), we show that mice form both affective and attentional sets and that their ability to shift away from these sets depends critically on the OFC and MFC regions. These results are identical to those reported in rats and similar to those reported in marmosets, providing a behavioral model in which to assess prefrontal function in mice.

\section{Materials and Methods}

Animals. Adult male C57BL/6J mice ( $>12$ weeks old) were purchased from The Jackson Laboratory. Experiments were conducted in accordance with Institutional Animal Care and Use Committee (University of Maryland School of Medicine, Baltimore, MD)-approved protocols and the Policies on the Use of Animals and Humans in Neuroscience Research. Under sterile conditions, bilateral stereotaxic lesions were made in MFC [anteroposterior (AP), $1.9 \mathrm{~mm}$; mediolateral $(\mathrm{ML}), \pm 0.3 \mathrm{~mm}$; ventral $(\mathrm{V}), 3.2 \mathrm{~mm}$ ] or in OFC regions (AP, $2.6 \mathrm{~mm}$; ML, $\pm 1.2 \mathrm{~mm} ; \mathrm{V}, 2.8 \mathrm{~mm}$ ) of anesthetized (isoflurane) mice using established coordinates (Paxinos and Franklin, 2001). At each lesion site, $\sim 0.1 \mu \mathrm{l}$ of sterile NMDA (12.5 $\mathrm{mg} / \mathrm{ml}$ in $0.9 \%$ saline; Sigma) was injected using a pulled glass pipette and a picospritzer. For control sham lesions, saline vehicle alone was injected. Each group was tested on the reversal/set-shift task after a 2 week recovery period.

Immunohistochemistry. Mice were transcardially perfused using a $4 \%$ paraformaldehyde in sodium phosphate $(0.1 \mathrm{M}, \mathrm{pH} 7.2)$ fixative, cut on a sliding freezing microtome into coronal sections $(50 \mu \mathrm{m})$ and processed for immunohistochemical analysis according to standard laboratory protocols (Martins et al., 2007). Rabbit anti-glial fibrillary acidic protein (GFAP) antibody was used (1:2000 dilution; Sigma), in Tris-buffered saline with $0.3 \%$ Triton X-100 and 5\% lamb serum (Invitrogen). Appropriately conjugated secondary antibodies (Jackson ImmunoResearch) were used at a 1:6000 dilution. Sections were stained with cresyl violet to visualize cell nuclei and cytoarchitecture. Images were obtained with a Leica DMRX bright field microscope and assembled and labeled in Adobe Photoshop CS2. Extent of lesion was assessed by cresyl violet staining and affected regions were noted on scanned images from Paxinos and Franklin adult mouse brain atlas (Paxinos and Franklin, 2001).

Reversal/set-shift task. After recovery from surgery, mice were tested on a variant of the reversal/set-shifting task developed by Brown and colleagues for rats (Birrell and Brown, 2000; McAlonan and Brown, 2003) and adapted for mice (Colacicco et al., 2002; Garner et al., 2006) (Tables $1,2)$. Mice were food deprived to reduce body weight to $85 \%$ of the ad libitum feeding weight and habituated with testing materials for $3 \mathrm{~d}$ before evaluation.

At the start of each trial, the mouse was placed in the testing arena to explore two bowls with combinations of odors and digging media until digging in one bowl to signify a choice. The bait was a piece of Honey Nut
Cheerios cereal ( $\sim 5 \mathrm{mg})$, and the cues, either olfactory (odor) or somatosensory and visual (texture of the digging medium which hides the bait), were altered and counterbalanced. All cues were presented in identical small animal food bowls (All Living Things Nibble bowls; PetSmart) that were identical in color and size. Digging media were mixed with the odor $(0.01 \%$ by volume $)$ and Honey Nut Cheerio powder $(0.1 \%$ by volume). All odors were ground dried spices (Penzeys, Hershey Chocolate, or McCormick), and unscented digging media was purchased from PetSmart [KayKob, bedding, wood chips, aquarium gravel, aquarium stone, kitty litter (two types)] or local discount stores (cotton balls, feathers, moss, plastic pellets, shredded paper, perlite, bark, packing peanuts). The mice were housed in Softcard bedding. The digging media did not contain any components used in the animal bedding. On the first day of training, the mice were given four consecutive trials with the baited food bowl to ascertain they could reliably dig. All mice were able to dig for the reward. The testing was performed over a $4 \mathrm{~d}$ period.

Mice were tested through a series of discriminations where the exemplar pair was changed, but the dimension (odor or medium) of the correct choice remained the same. The dimension was relevant if its attributes predicted outcome. For example, if odor was the relevant dimension, then the mouse was required to choose the correct odor from each pair and ignore the attributes of the digging medium. In this example, the digging medium is considered the irrelevant dimension.

The discriminations (Tables 1,2) were as follows: (1) a single series of simple discriminations (SDs) in which the mouse was presented with two choices of the relevant dimension and one choice of the irrelevant dimension (i.e., two odors within the same medium); (2) a single series of compound discriminations (CDs) in which the mouse was presented with the same choices of relevant dimension as in the SD and two choices of irrelevant dimensions (the exemplar used in the SD and a new exemplar); (3) a series of four intradimensional shifts (IDSs I-IV) in which the mouse was presented with compound discriminations using two novel exemplars from the relevant and irrelevant dimensions for each IDS (the relevant dimension of the correct choice (i.e., odor) was maintained throughout the discriminations); (4) a reversal discrimination (IDS IVrev) in which the mouse was presented with the same set of exemplars as in IDS IV, but the stimulus-reward pairing was reversed within the relevant dimension; and (5) an extradimensional shift (EDS) in which the mouse was presented with a novel compound discrimination, except for the first time the correct choice was an exemplar that was previously from the irrelevant dimension (the previously relevant dimension has become irrelevant).

The baited bowl was randomly presented on either side of the testing cage, and the relevant exemplar was randomly presented with the irrelevant exemplars. The trial was stopped if the mouse did not dig within 3 $\min$ in the testing cage. Stopped trials were uncommon $(<3 \%$ of all trials), and they occurred most frequently during the SD. Aborted trials were not observed after completion of the $\mathrm{CD}$ and were not included in the latency calculations. The order of discriminations and exemplars was the same for all mice, but the direction of the EDS (odor to medium or medium to odor) was counterbalanced within each experimental group. A criterion of eight consecutive correct trials was required to complete each task. Data are reported as the number of trials to criterion and the number of errors required for each discrimination.

Data analysis. Values are reported as the mean \pm SEM. For trials to criteria and errors, a two-way ANOVA was used to determine statistical significance between treatment groups and discriminations, followed by Fisher least-significant difference post hoc analysis. Analysis was performed with the Statistica software package (Statsoft). Statistical significance was considered as $p<0.05$, and denoted by asterisks in the figures. 
Table 2. Exemplar combinations

\begin{tabular}{lcll}
\hline & & Dimension & \\
\cline { 3 - 4 } Pair & Exemplar & Odor & Medium \\
\hline 1 & 1 & Rosemary & Aspen bedding \\
& 2 & Cloves & Gravel \\
2 & 3 & Cinnamon & Kaykob bedding \\
& 4 & Sage & Moss \\
4 & 5 & Onion & Perlite \\
& 6 & Paprika & Bark \\
& 7 & Garlic & Cat litter \\
& 8 & Coriander & Feathers \\
& 9 & Thyme & Plastic pellets \\
6 & 10 & Black pepper & Cotton balls \\
& 11 & Cumin & Shredded paper \\
& 12 & Cardamom & Packing peanut pieces \\
\hline
\end{tabular}

\section{Results}

Area-specific lesions impair reversal and set-shifting ability

Lesions

Studies in rats and primates show that reversal learning and set shifting reflect functions in specific prefrontal subdivisions. To assess parallel functioning in the murine prefrontal cortex, the OFC or MFC regions, as anatomically defined (Paxinos and Franklin, 2001), were lesioned with NMDA. Sham-lesioned control mice received infusions of saline vehicle. The neurotoxic lesions were characterized by anatomical methods. Of the group of 10 mice receiving NMDA lesions targeted to the OFC area, 8 had damage within the OFC area. For the MFC lesion group, 8 of 10 mice had selective damage within the medial wall. Representations of the lesioned areas and the range of the extent of damage for these mice are shown in Figure 1, $A$ and $B$. Cresyl violet histology demonstrated cell loss in both OFC (Fig. 1C,D) and MFC (Fig. $1 F, G$ ) areas. Immunoreactivity for the gliotic scar marker GFAP was observed in the lesioned areas (Fig. $1 E, H$ ).

\section{Reversal set-shifting task}

Mice readily performed the discriminations, as described in previous rat studies (Birrell and Brown, 2000; McAlonan and Brown, 2003), indicating that mice respond to the rules set forth by these exemplars (Fig. 2A). ANOVA indicated main effects of group $\left(F_{(2,144)}=3.86, p=0.0232\right)$ and task $\left(F_{(7,144)}=12.92, p<\right.$ $0.0001)$. All groups learned the problems; however, the MFClesioned group required more trials to reach criterion on the SD [compare $13.5 \pm 1.9$ (MFC area) with $10.6 \pm 1.0$ (sham) and $10.3 \pm 1.2$ (OFC area), $p=0.0017$, for both post hoc comparisons]. No differences among groups were found in the rest of the training discriminations (CD-IDS IV; $p>0.39$ ). The relevant dimension, either odor or medium, was counterbalanced within the experimental subjects, and the same results were obtained when dimension was considered as a variable $\left(F_{(1,144)}, p=0.81\right)$, suggesting equivalent valence for both odor and medium. However, a decrease in response latency was observed as the discriminations proceeded, and the response latency for the IDS III was significantly less than the SD and CD latencies (supplemental Fig. $\mathrm{S} 1$, available at www.jneurosci.org as supplemental material).

Mice had more difficulty meeting criteria when the associations were reversed (Fig. 2A, IDS IVrev). Sham mice demonstrated reversal learning, by the increased numbers of trials required for the IDS IVrev $(15.6 \pm 1.7)$ compared with IDS IV $(9.5 \pm 0.8, p<0.0001)$ (Fig. $2 A)$. Mice were able to perform multiple reversal discriminations, and showed increased trials to criterion when as many as three reversals were tested (supplemental Table S1, Fig. S2, available at www.jneurosci.org as sup- plemental material). Neurotoxic damage to the murine OFC area impaired ability to reach criterion on reversal associations [compare $22.4 \pm 2.0$ trials (OFC area) for IDS IVrev, $p=0.0017$ ) (Fig. $2 A)$. The effect was specific to the OFC area, as mice with MFC lesions performed similarly to sham animals $(16.3 \pm 2.3$ trials, $p=0.6959)$, but were significantly different from the OFClesioned group $(p=0.0057)$.

To test attentional set shifting, the reward-predicting dimension was changed, from odor to medium (or vice versa) in the EDS discrimination. Sham mice demonstrated an increase in trials necessary to achieve criterion, comparing the IDS IV (9.5 \pm $0.8)$ (Fig. $2 A)$ and the EDS $(13.0 \pm 0.8, p<0.002)$. OFC-lesioned mice were similar to the sham group $(13.1 \pm 1.3$ trials, $p=0.65)$ However, the formation of the attentional set was impaired in the MFC-lesioned subjects, as the MFC-lesioned mice needed $22.3 \pm$ 2.4 trials to complete the EDS ( $p<0.0003$ compared with either sham or OFC).

We also compared the numbers of errors for each task (Fig. $2 B)$. Although there were effects of problem $\left(F_{(7,143)}=27.7, p<\right.$ 0.0001 ), post hoc comparisons showed no differences between groups in errors made during training phase (SD-IDS IV). In agreement with the trials needed for criterion, the number of errors to complete the reversal task increased significantly from $0.8 \pm 0.4$ (IDS IV) to $5.1 \pm 1.1$ (IDS IVrev, $p<0.0001$ ) in the sham mice. As expected, the number of errors made by the OFClesioned mice was greater than the sham and MFC groups (OFC, $7.6 \pm 1.1$ errors; sham, $5.1 \pm 1.1$ errors; and MFC, $3.43 \pm 0.78$ errors). Post hoc analysis indicated that the difference in errors between the sham and OFC groups was significant ( $p=0.0021$ ), but not between the sham and MFC groups. In summary, only the OFC-lesioned group made more errors on the reversal task, indicating that mouse OFC area contributes to the formation of affective learning sets.

For the sham group, the number of errors also increased significantly for the EDS discrimination from $0.8 \pm 0.4$ (IDS IV) to $(2.1 \pm 0.4, p<0.02)$, indicating set shifting. The numbers of errors between sham $(2.1 \pm 0.4)$ and OFC $(1.9 \pm 0.5)$ groups were the same for the EDS task ( $p=0.4614)$. The errors made by the MFC-lesioned mice were different from the sham and OFClesioned groups on the set-shifting task (Fig. 2B) (EDS, $p=$ 0.0003 ). These data indicate that lesions to the MFC area selectively alter formation of the attentional set and impair shifting between dimensions.

Set shifting is dependent on number of tasks and overtraining In the lesion experiments (Fig. $2 A$ ), the mice were presented with seven discriminations (CD-IDS IVrev) with the same relevant dimension before being challenged by the set-shifting rule. This paradigm demonstrated successful set-shifting ability. However, literature reports with fewer discriminations suggested that mice were unable to form attentional sets (Colacicco et al., 2002), and that overtraining may improve set-shifting ability (Garner et al., 2006). Initially, we performed the task as outlined in the literature (Birrell and Brown, 2000; Colacicco et al., 2002), and the mice demonstrated reversal learning (supplemental Fig. S2, available at www.jneurosci.org as supplemental material), but not formation of the attentional set. Examination of the trial and latency data indicated that the mice did not learn in the same manner as rats, leading us to further examine multiple components of the task. We systematically examined the effects of task number, presence and location of reversals, and inclusion of overtraining on set-shifting ability in mice (supplemental Table S2, available at www.jneurosci.org as supplemental material). To compare ex- 
A

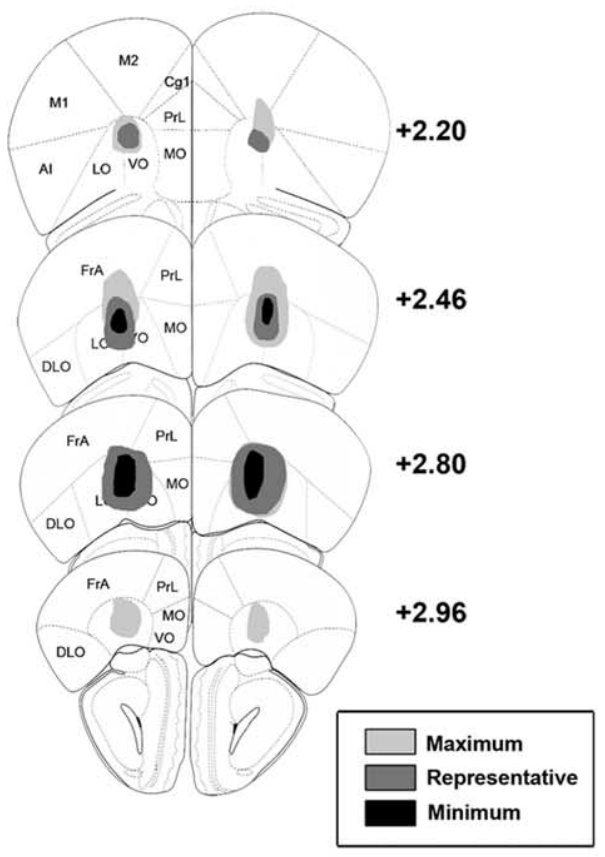

B

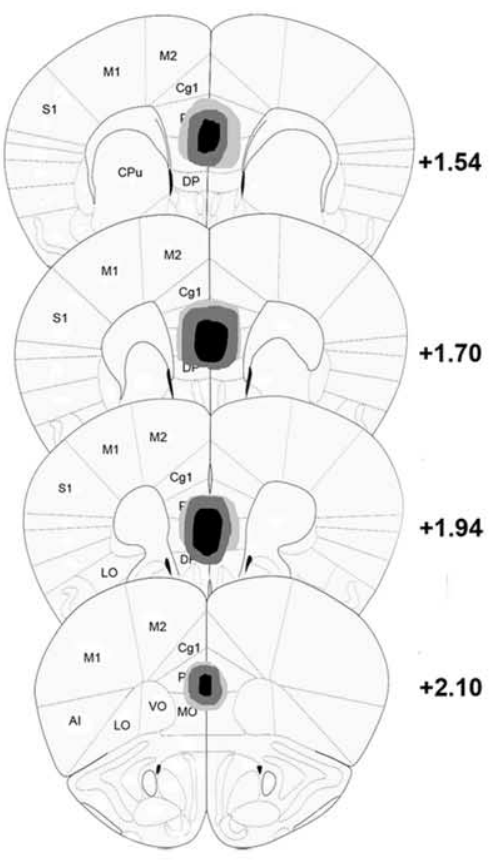

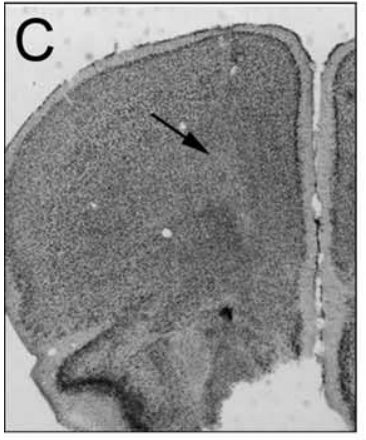

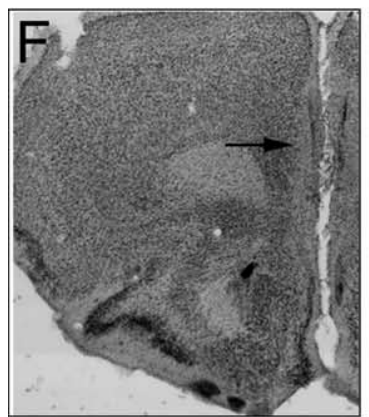

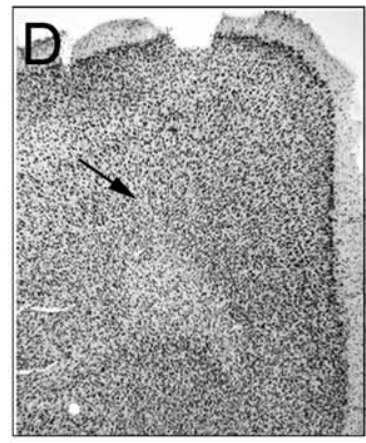

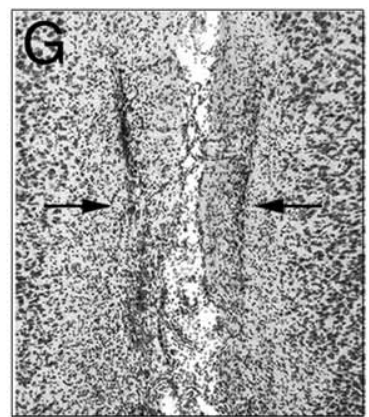

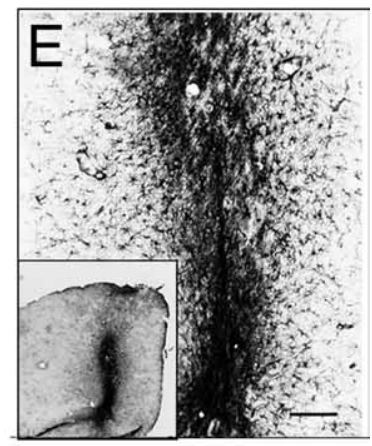

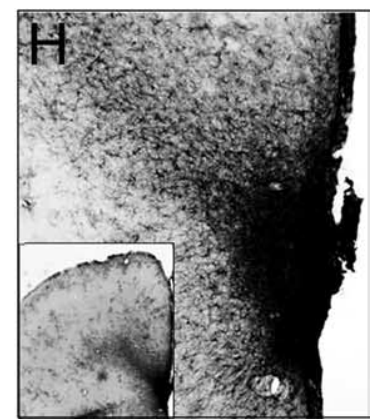

Figure 1. Lesions demonstrate selective impairments in reversal learning and set-shifting ability. $\boldsymbol{A}, \boldsymbol{B}$, Distribution of lesioned areas. Cresyl violet staining was used to determine lesioned regions. The maximum extent of lesions is denoted by the lightest gray shading, and the minimum extent of lesions in all mice is shown by black shading. Representative affected areas, present in at least $50 \%$ of the subjects, are shown by medium gray. The figures represent $n>7$ mice per group; no significant damage was observed in sections from the sham-lesion mice. Drawings were adapted from the atlas of Paxinos and Franklin (2001). C, D, Cresyl-violet-stained sections with OFC lesions. Arrows point to lesion area. E, GFAP immunoreactivity demonstrates gliosis at the OFC lesion site; the inset shows the location of the lesion in low power image. $\boldsymbol{F}, \boldsymbol{G}$, Cresyl-violet-stained sections of MFC lesions. In these images, bilateral lesions are shown by the arrows. $\boldsymbol{H}$, GFAP immunoreactivity demonstrates gliosis at the MFC lesion site. Scale bar: $\boldsymbol{C}, \boldsymbol{F}, 500 \mu \mathrm{m} ; \boldsymbol{D}, \boldsymbol{G}, 400 \mu \mathrm{m} ; \boldsymbol{E}, \boldsymbol{H}, 200 \mu \mathrm{m}$.

perimental groups, we calculated the ratio of trials needed for criterion for the EDS to those needed for the preceding IDS (Fig. $3)$. The main effect was observed with task number $\left(F_{(2,32)}=\right.$ $65.4, p<0.0001)$. The presence or location of reversal discrimi- nations had no effect on set-shifting ability. The addition of overtraining increased the ratio of trials (group 3, $1.72 \pm 0.23$ ) (Fig. 3), but this increase was not significant (compared with group 2, $1.44 \pm 0.14$, $p=0.25$ ). Therefore, mouse set-shifting ability is dependent on the number of previously encountered discrimination problems within the same dimension. In summary, these experiments yielded outcomes that suggest that mice form affective and attentional sets to solve problems.

\section{Discussion}

This study demonstrates that mice, like rats and primates, are capable of forming affective and attentional sets (Dias et al., 1996a). Thus, sham control mice exhibited significant increases in the trials to acquire reversal learning and also shift attention between learning rules. These increases in trials show that the mice, like other species, attempt to learn rules to allow them to generalize from one problem to the next. When these rules are violated, by changes either in a previously acquired problem or attentional set, then mice require more trials to successfully complete the new problem, because they must overcome the influence of these normally helpful rules. Interestingly, in agreement with Garner et al. (2006), mice appear to form these rules less efficiently than rats, and therefore additional presentations of similar problems are required to strengthen the formation of the attentional set. The murine response to rule changes is directly analogous to the difficulty experienced by rats and primate species when presented with reversal problems or set shifts in similar tasks.

Furthermore, data presented in the current report show that, like rats and primates, the ability to overcome these rules depends in part on subdivisions within the frontal pole of the murine brain. Lesions to the OFC area caused a selective deficit in reversal learning, indicating that the OFClesioned mice had more trouble than normal overcoming the affective rule. In contrast, lesions to the medial wall (MFC area) caused a selective deficit in set shifting, indicating that the MFC-lesioned mice had more trouble than normal switching the attentional rule. These results are identical to what has been reported in rats, where damage to the OFC and MFC areas causes a double dissociation in impaired reversal learning and set shifting, respectively (Birrell and Brown, 2000; McAlonan and Brown, 2003). Similarly in marmosets, it has been shown that OFC lesions impair reversal learning, whereas damage to lateral prefrontal cortex disrupts set shifting (Dias et al., 1996a,b; Clarke et al., 2005). Although rodent frontal 


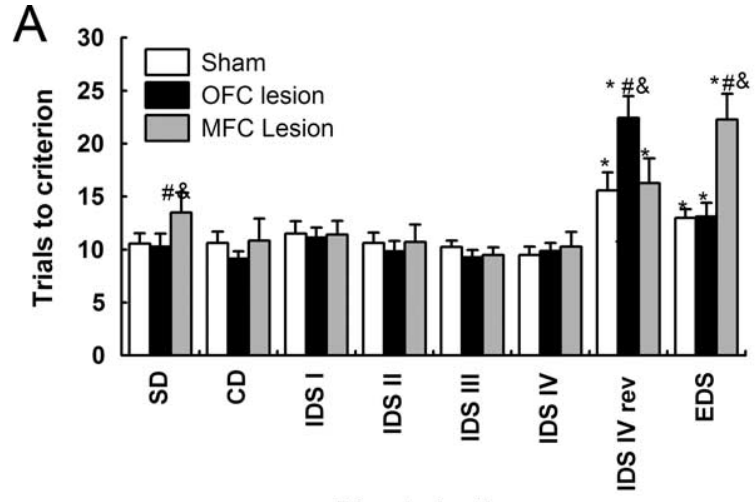

Discrimination

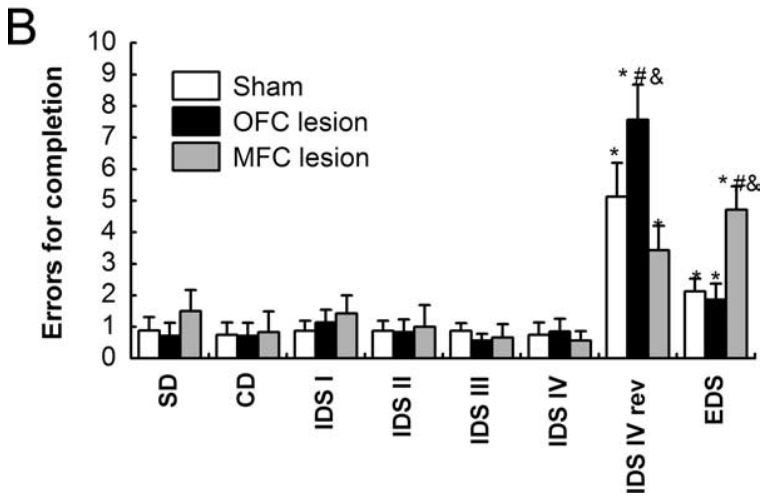

Figure 2. Lesions selectively impair performance on reversal and set-shifting tasks. $A$, The number of trials to reach criterion is the same for training, whereas more trials are needed for the reversal learning (IDS IVrev) and for the ID-ED shift (EDS). The OFC-lesion group required more trials to complete the reversal task (IDS IVrev), and the MFC lesion required more trials for the ID-ED shift. *Significant difference between IDS IV and either IDS IVrev or EDS; "difference between control sham group and either the OFC- or MFC-lesioned group for the specific discrimination; ${ }^{\circledR}$ difference between the MFC-and OFC-lesioned groups for the specific discrimination. Significance is $p<0.05$. Bars represent groups of $n>7$ mice per group. $\boldsymbol{B}$, The numbers of errors recorded were similar between all groups during the learning phase. The OFC lesion group had significantly more errors than the sham group $\left({ }^{\#} p<0.05\right)$ or the MFC group $\left({ }^{\&} p<0.05\right)$ for the IDS IVrev task, whereas the MFC lesion group was similar to the sham group for the IDS IVrev. The MFC lesion group had significantly more errors on the EDS task, compared with the sham group ( ${ }^{\#} p<0.05$ ) or the $0 \mathrm{FC}$ group $\left({ }^{\&} p<0.05\right)$. OFC lesions did not affect performance on the EDS task.

areas do not share the anatomical complexities of the primate, these areas are defined based on similarities in connectivity and function (Guldin et al., 1981; Uylings et al., 2003). The behavioral deficits in affective and attentional sets exhibited by the mouse after circumscribed lesions to the frontal pole imply that the murine brain shares a subset of rule acquisition and problem solving abilities with the rat and primate brain. The availability of a mouse model enables rapid analysis of the cognitive consequences of genetic and developmental manipulations responsible for human neuropsychiatric disorders.

Our studies demonstrated that a single IDS discrimination is insufficient to form the attentional set in mice. However, training on multiple discriminations and exemplar sets with the same dimension yields consistent formation of the attentional set; additional IDS tests did not improve formation of the attentional set. The need for multiple discriminations is supported by response latency data that demonstrates a significant decrease only after four discriminations. This requirement may explain why our results differ from two previous reports, which have used such brief training procedures and reported an inability to generate attentional sets in mice (Colacicco et al., 2002; Brigman et

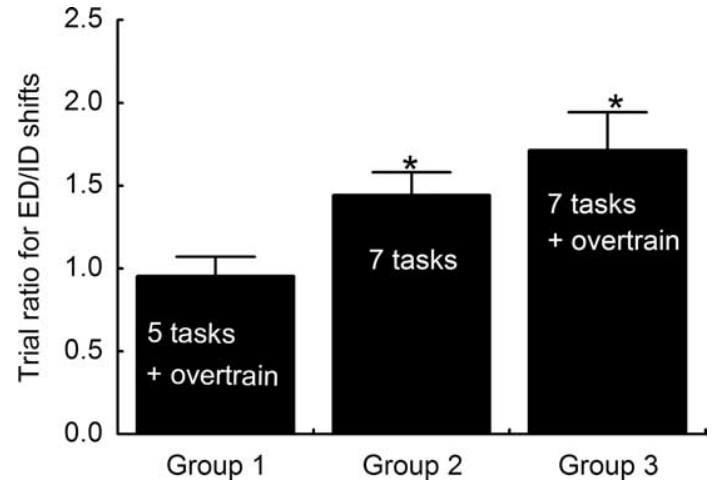

Figure 3. We systematically examined the effects of task number, presence and location of reversals, and inclusion of overtraining on set-shifting ability in mice (supplemental Table S2, available at www.jneurosci.org as supplemental material). To compare experimental groups, we calculated the ratio of trials needed for criterion for the EDS to those needed for the preceding IDS. In group 1, five separate tasks were insufficient to form as attentional set, as indicated by a ratio of ED/ID trials of 1 . In groups 2 and 3 , seven tasks were sufficient to form an attentional set. The main effect was observed with task number $\left(F_{(2,32)}=65.4, p<0.0001\right)$. The presence or location of reversal discriminations or overtraining had no effect on set-shifting ability. Asterisks denote a significant different from group $1(p<0.04)$.

al., 2005). Our results agree with Garner et al. (2006) that additional presentations of the same dimension strengthens the formation of the attentional set. The cohort of mice used by Garner et al. (2006) included a mix of males and females of varying ages with the task performed over several months, whereas the data presented in this report are male mice. The difference in effect of overtraining may be attributable to inclusion of the female subjects, as females were observed to respond to the discrimination problems significantly differently than their male counterparts (our unpublished observation). Overall, mice perform the tasks similarly, but not identically, to their rat counterparts.

Here, the mice required the same range of numbers of trials on the discriminations to reach criterion as reported for rats (Birrell and Brown, 2000; McAlonan and Brown, 2003) and C57BL/6 mice from different sources (Colacicco et al., 2002; Garner et al., 2006). The exemplars of texture and odor are easily discriminated by the rodents and require few trials over criterion to learn the initial training problems in contrast to two visual cues (Brigman et al., 2005, 2006; Izquierdo et al., 2006). In many reported versions of this task, learning does not appear to be reflected by decreasing trials to criterion as more discriminations are presented (Birrell and Brown, 2000; Colacicco et al., 2002; McAlonan and Brown, 2003; Tunbridge et al., 2004; Glickstein et al., 2005; Black et al., 2006; Lapiz and Morilak, 2006). However, a decrease in latency to choice was used as an indicator of improved performance (Colacicco et al., 2002). Our data demonstrated a similar decrease. Thus, our mice performed the task similarly to rats and demonstrated learning by two measures: first, the decreased latency to choice on multiple consecutive compound discriminations (CD-IDS IV), and second, the increased trials for the reversal and set-shifting discriminations.

The MFC-lesioned group demonstrated impaired learning on the first discrimination (SD), but similar performance on subsequent training discriminations. These results are in agreement with rat lesion studies using the continuous spatial-delayed alternation task (Schwabe et al., 2004), and in instrumental conditioning (Ostlund and Balleine, 2005). Data with MFC-lesioned rats on the eight arm-radial maze (McDonald et al., 2007) and on this reversal set-shifting task after cocaine administration in rats (Black et al., 2006) show a similar trend, suggesting that impaired 
MFC function can delay acquisition of a task. Thus, the MFClesioned mice appeared to have delayed acquisition of the initial discrimination, but eventually learned the task, as the numbers of trials to criterion and response latencies were the same as sham controls in later discriminations.

The order of the presentation of the reversal task did not alter the ability to form the attentional set, also in agreement with the overtraining concept that the EDS is dependent on the repeated presentations of the relevant dimensions (Garner et al., 2006). Several details of our test are slightly different from previous reports, including different exemplar pairs, because of availability or response. All of our materials were tested for equivalent valence, independent of the type of discrimination. Sand and dirt, which are very naturalistic media, were avoided because the mouse stopped digging in the medium to clean its whiskers. However, these changes do not appear to significantly alter the ability of the mouse to perform the reversal learning or the set shifting. The key factor is the number of presentations of the discriminations to strengthen the learning rules.

Proper functioning of the prefrontal cortical areas is dependent on multiple neurotransmitter systems including catecholamines, serotonin, and GABA. Depletion of serotonin impairs OFC mediated reversal learning in nonhuman primates (Clarke et al., 2005), whereas loss of dopamine in the OFC area has no effect on reversal learning (Clarke et al., 2007). In the MFC area, imbalances in catecholamines, mainly dopamine, impaired set shifting in marmosets and rats (Tait et al., 2007; McGaughy et al., 2008; Newman et al., 2008). In humans, loss of dopamine, along with GABA, has been implicated in decreased working memory (Lewis et al., 2005; Hashimoto et al., 2008). Modulation of the balance of inhibitory to excitatory output appears to be critical in all species for proper prefrontal function (Wilson et al., 1994; Rao et al., 2000; Constantinidis et al., 2002; Schwabe et al., 2004; Tunbridge et al., 2004; Kim and Ragozzino, 2005; Black et al., 2006; Floresco et al., 2006; Lapiz and Morilak, 2006). Future studies will examine the genetic and developmental pathways that lead to long-term disruptions in prefrontal anatomy and function.

\section{References}

Birrell JM, Brown VJ (2000) Medial frontal cortex mediates perceptual attentional set shifting in the rat. J Neurosci 20:4320-4324.

Black YD, Maclaren FR, Naydenov AV, Carlezon WA Jr, Baxter MG, Konradi C (2006) Altered attention and prefrontal cortex gene expression in rats after binge-like exposure to cocaine during adolescence. J Neurosci 26:9656-9665.

Brigman JL, Bussey TJ, Saksida LM, Rothblat LA (2005) Discrimination of multidimensional visual stimuli by mice: intra- and extradimensional shifts. Behav Neurosci 119:839-842.

Brigman JL, Padukiewicz KE, Sutherland ML, Rothblat LA (2006) Executive functions in the heterozygous reeler mouse model of schizophrenia. Behav Neurosci 120:984-988.

Chudasama Y, Robbins TW (2003) Dissociable contributions of the orbitofrontal and infralimbic cortex to pavlovian autoshaping and discrimination reversal learning: further evidence for the functional heterogeneity of the rodent frontal cortex. J Neurosci 23:8771-8780.

Clarke HF, Dalley JW, Crofts HS, Robbins TW, Roberts AC (2004) Cognitive inflexibility after prefrontal serotonin depletion. Science 304:878-880.

Clarke HF, Walker SC, Crofts HS, Dalley JW, Robbins TW, Roberts AC (2005) Prefrontal serotonin depletion affects reversal learning but not attentional set shifting. J Neurosci 25:532-538.

Clarke HF, Walker SC, Dalley JW, Robbins TW, Roberts AC (2007) Cognitive inflexibility after prefrontal serotonin depletion is behaviorally and neurochemically specific. Cereb Cortex 17:18-27.

Colacicco G, Welzl H, Lipp HP, Würbel H (2002) Attentional set-shifting in mice: modification of a rat paradigm, and evidence for strain-dependent variation. Behav Brain Res 132:95-102.

Constantinidis C, Williams GV, Goldman-Rakic PS (2002) A role for inhibition in shaping the temporal flow of information in prefrontal cortex. Nat Neurosci 5:175-180.

Dias R, Robbins TW, Roberts AC (1996a) Dissociation in prefrontal cortex of affective and attentional shifts. Nature 380:69-72.

Dias R, Robbins TW, Roberts AC (1996b) Primate analogue of the Wisconsin Card Sorting Test: effects of excitotoxic lesions of the prefrontal cortex in the marmoset. Behav Neurosci 110:872-886.

Floresco SB, Magyar O, Ghods-Sharifi S, Vexelman C, Tse MT (2006) Multiple dopamine receptor subtypes in the medial prefrontal cortex of the rat regulate set-shifting. Neuropsychopharmacology 31:297-309.

Garner JP, Thogerson CM, Würbel H, Murray JD, Mench JA (2006) Animal neuropsychology: validation of the Intra-Dimensional ExtraDimensional set shifting task for mice. Behav Brain Res 173:53-61.

Glickstein SB, Desteno DA, Hof PR, Schmauss C (2005) Mice lacking dopamine D2 and D3 receptors exhibit differential activation of prefrontal cortical neurons during tasks requiring attention. Cereb Cortex 15:1016-1024

Goldstein B, Obrzut JE, John C, Ledakis G, Armstrong CL (2004) The impact of frontal and non-frontal brain tumor lesions on Wisconsin Card Sorting Test performance. Brain Cogn 54:110-116.

Guldin WO, Pritzel M, Markowitsch HJ (1981) Prefrontal cortex of the mouse defined as cortical projection area of the thalamic mediodorsal nucleus. Brain Behav Evol 19:93-107.

Hashimoto T, Arion D, Unger T, Maldonado-Avilés JG, Morris HM, Volk DW, Mirnics K, Lewis DA (2008) Alterations in GABA-related transcriptome in the dorsolateral prefrontal cortex of subjects with schizophrenia. Mol Psychiatry 13:147-161.

Izquierdo A, Wiedholz LM, Millstein RA, Yang RJ, Bussey TJ, Saksida LM, Holmes A (2006) Genetic and dopaminergic modulation of reversal learning in a touchscreen-based operant procedure for mice. Behav Brain Res 171:181-188.

Kim J, Ragozzino ME (2005) The involvement of the orbitofrontal cortex in learning under changing task contingencies. Neurobiol Learn Mem 83:125-133.

Kolb B (1984) Functions of the frontal cortex of the rat: a comparative review. Brain Res 320:65-98.

Lapiz MD, Morilak DA (2006) Noradrenergic modulation of cognitive function in rat medial prefrontal cortex as measured by attentional set shifting capability. Neuroscience 137:1039-1049.

Lewis DA, Hashimoto T, Volk DW (2005) Cortical inhibitory neurons and schizophrenia. Nat Rev Neurosci 6:312-324.

Lidow MS, Koh PO, Arnsten AF (2003) D1 dopamine receptors in the mouse prefrontal cortex: Immunocytochemical and cognitive neuropharmacological analyses. Synapse 47:101-108.

Martins GJ, Plachez C, Powell EM (2007) Loss of embryonic MET signaling alters profiles of hippocampal interneurons. Dev Neurosci 29:143-158.

McAlonan K, Brown VJ (2003) Orbital prefrontal cortex mediates reversal learning and not attentional set shifting in the rat. Behav Brain Res 146:97-103.

McDonald RJ, Foong N, Ray C, Rizos Z, Hong NS (2007) The role of medial prefrontal cortex in context-specific inhibition during reversal learning of a visual discrimination. Exp Brain Res 177:509-519.

McGaughy J, Ross RS, Eichenbaum H (2008) Noradrenergic, but not cholinergic, deafferentation of prefrontal cortex impairs attentional setshifting. Neuroscience 153:63-71.

Nelson HE (1976) A modified card sorting test sensitive to frontal lobe defects. Cortex 12:313-324.

Newman LA, Darling J, McGaughy J (2008) Atomoxetine reverses attentional deficits produced by noradrenergic deafferentation of medial prefrontal cortex. Psychopharmacology (Berl) 200:39-50.

O’Doherty J, Critchley H, Deichmann R, Dolan RJ (2003) Dissociating valence of outcome from behavioral control in human orbital and ventral prefrontal cortices. J Neurosci 23:7931-7939.

Ostlund SB, Balleine BW (2005) Lesions of medial prefrontal cortex disrupt the acquisition but not the expression of goal-directed learning. J Neurosci 25:7763-7770.

Pantelis C, Barber FZ, Barnes TR, Nelson HE, Owen AM, Robbins TW (1999) Comparison of set-shifting ability in patients with chronic schizophrenia and frontal lobe damage. Schizophr Res 37:251-270. 
Paxinos G, Franklin KBJ (2001) The mouse brain in stereotaxic coordinates, ed 2. San Diego: Academic.

Preuss TM (1995) Do rats have a prefrontal cortex? The Rose-WoolseyAkert Program reconsidered. J Cogn Neurosci 7:1-24.

Rao SG, Williams GV, Goldman-Rakic PS (2000) Destruction and creation of spatial tuning by disinhibition: GABA(A) blockade of prefrontal cortical neurons engaged by working memory. J Neurosci 20:485-494.

Remijnse PL, Nielen MM, Uylings HB, Veltman DJ (2005) Neural correlates of a reversal learning task with an affectively neutral baseline: an eventrelated fMRI study. Neuroimage 26:609-618.

Schoenbaum G, Roesch M (2005) Orbitofrontal cortex, associative learning, and expectancies. Neuron 47:633-636.

Schoenbaum G, Setlow B, Ramus SJ (2003a) A systems approach to orbitofrontal cortex function: recordings in rat orbitofrontal cortex reveal interactions with different learning systems. Behav Brain Res 146:19-29.

Schoenbaum G, Setlow B, Nugent SL, Saddoris MP, Gallagher M (2003b) Lesions of orbitofrontal cortex and basolateral amygdala complex disrupt acquisition of odor-guided discriminations and reversals. Learn Mem 10:129-140.

Schwabe K, Enkel T, Klein S, Schütte M, Koch M (2004) Effects of neonatal lesions of the medial prefrontal cortex on adult rat behaviour. Behav Brain Res 153:21-34.

Shad MU, Tamminga CA, Cullum M, Haas GL, Keshavan MS (2006) In- sight and frontal cortical function in schizophrenia: a review. Schizophr Res 86:54-70.

Tait DS, Brown VJ, Farovik A, Theobald DE, Dalley JW, Robbins TW (2007) Lesions of the dorsal noradrenergic bundle impair attentional set-shifting in the rat. Eur J Neurosci 25:3719-3724.

Thoma P, Wiebel B, Daum I (2007) Response inhibition and cognitive flexibility in schizophrenia with and without comorbid substance use disorder. Schizophr Res 92:168-180.

Tunbridge EM, Bannerman DM, Sharp T, Harrison PJ (2004) Catechol-omethyltransferase inhibition improves set-shifting performance and elevates stimulated dopamine release in the rat prefrontal cortex. J Neurosci 24:5331-5335.

Uylings HB, Groenewegen HJ, Kolb B (2003) Do rats have a prefrontal cortex? Behav Brain Res 146:3-17.

Verdejo-García A, Bechara A, Recknor EC, Pérez-García M (2006) Executive dysfunction in substance dependent individuals during drug use and abstinence: an examination of the behavioral, cognitive and emotional correlates of addiction. J Int Neuropsychol Soc 12:405-415.

Wilson FA, O'Scalaidhe SP, Goldman-Rakic PS (1994) Functional synergism between putative gamma-aminobutyrate-containing neurons and pyramidal neurons in prefrontal cortex. Proc Natl Acad Sci U S A 91: 4009-4013. 